\title{
Groove pancreatitis: A common condition that is uncommonly diagnosed preoperatively
}

\author{
Rakesh Gupta BA ${ }^{1}$, Geoffrey S Williams MD FRCPC ${ }^{2}$, Valerie Keough MD FRCPC ${ }^{3}$
}

\section{CASE PRESENTATION}

A 47-year-old woman presented with a two-week history of severe right upper-quadrant abdominal pain radiating to the back. She reported no jaundice, weight loss of $4.5 \mathrm{~kg}$ over six months, no history of alcohol consumption, and no family history of pancreatic disease or autoimmune disease. Her symptoms briefly subsided then resumed two months later, prompting investigation.

Abdominal ultrasound revealed a dilated common bile duct (CBD) with a maximum diameter of $10 \mathrm{~mm}$ and a possible hypoechoic lesion arising from the pancreatic head, raising the possibility of a neoplasm (Figure 1). Computed tomography (CT) scan revealed two calcifications in the pancreatic head within the distal pancreatic duct, consistent with a pancreatic mass, along with stricture of the distal CBD and enlarged regional lymph nodes (Figure 2). Bloodwork revealed elevated alkaline phosphatase (196 U/L) and alanine aminotransferase (139 U/L) levels, normal total bilirubin levels and a mildly elevated CA 19-9 tumour marker level (42.5 kU/L). Subsequent referral for endoscopic retrograde cholangiopancreatography (ERCP) revealed a smooth, $2 \mathrm{~cm}$ stricture of the distal CBD; brush cytology was negative for malignant cells. A $9 \mathrm{~cm}, 10$ Fr plastic biliary stent was placed across the stricture and the patient experienced resolution of her abdominal pain.

It was believed that this was a benign distal CBD stricture; therefore, further workup was performed for causes of pancreatitis. The immunoglobulin G4 level was normal, as was the antinuclear antibody level. Metabolic workup, including triglycerides and calcium, was normal. Magnetic resonance imaging (MRI) of abdomen and pelvis revealed multiple, variable size cystic lesions, the largest measuring up to $1.6 \mathrm{~cm}$ and seen in close relation to the medial duodenal wall, along with bulkiness of appearance of the pancreatic head and uncinate process, and no definite pancreatic or ductal masses identified (Figure 3). Repeat ERCP performed seven weeks after the initial ERCP showed persistent smooth stricture of the distant CBD consistent with chronic pancreatitis (Figure 4). Again, cytology was negative for malignant cells. Following a hepatobiliary surgical consultation, an endoscopic ultrasound (EUS) with fine-needle aspiration was performed, revealing a hypoechoic, heterogeneous pancreatic head with two small cysts between the duodenal wall and pancreatic head, with associated parenchymal calcifications in the pancreatic head. The pancreatic body and tail appeared normal with normal pancreatic duct. Fine-needle aspiration was negative for malignant cells.

The MRI was then reviewed again with an expert in gastrointestinal radiographic imaging. The radiologist believed that the imaging characteristics were highly suggestive of groove pancreatitis (GP). The patient underwent two additional ERCPs in an attempt to dilate the distal CBD stricture. The attempts at dilation were unsuccessful and she was subsequently referred for surgical management of the stricture.

The patient was scheduled for exploratory laparotomy pancreaticoduodenectomy, but intraoperative exploration revealed an extremely hard, chronically inflamed gland that was better treated by

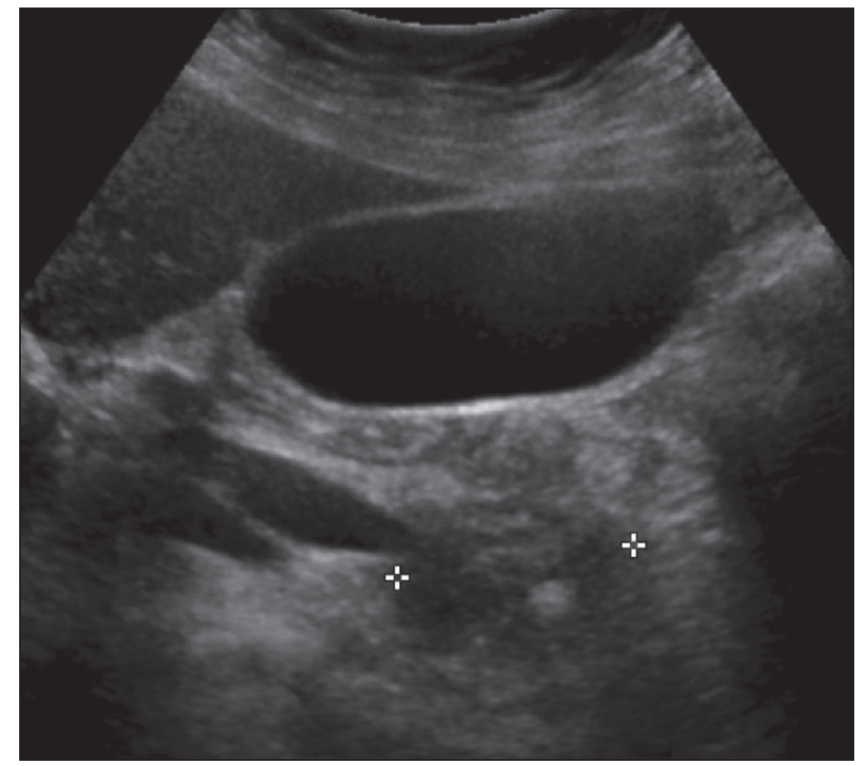

Figure 1) Ultrasound image demonstrating hypodense enlargement of the pancreatic head and regular marked tapering compatible with a stricture of the distal common bile duct. An echogenic focus in the pancreatic head is compatible with a calcification

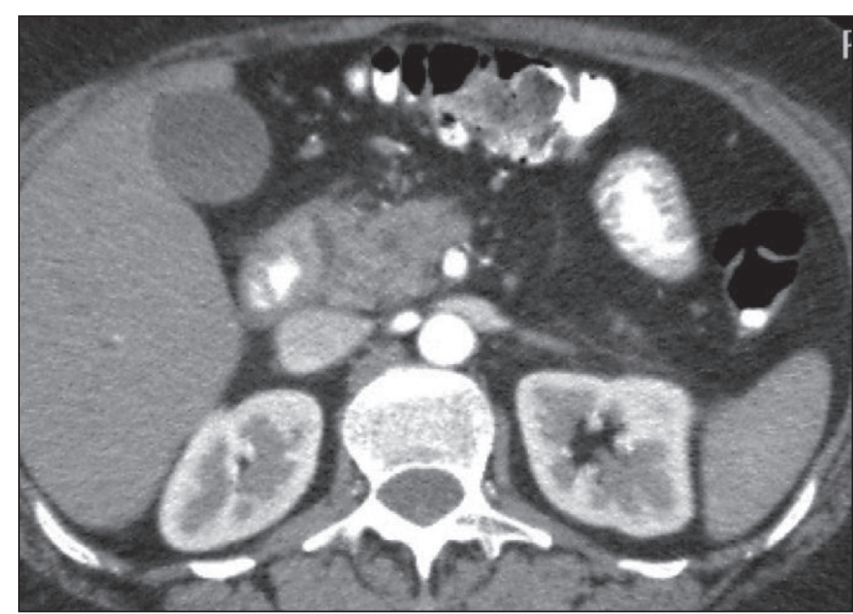

Figure 2) Image from contrast-enhanced computed tomography demonstrating increased soft tissue within the pancreaticoduodenal groove, thickening of the wall of the duodenum and fluid attenuation within the thickened duodenal wall. The pancreatic head is heterogeneous and contains calcifications. There is a tight regular stricture of the distal common bile duct

${ }^{1}$ Dalhousie Medical School; 2 Division of Gastroenterology, Department of Medicine; ${ }^{3}$ Department of Radiology, Dalhousie University, Halifax, Nova Scotia Correspondence: Dr Geoffrey S Williams, Division of Gastroenterology, Department of Medicine, Dalhousie University, Room 920, Victoria General

Hospital, 1276 Southpark Street, Halifax, Nova Scotia B3H 2Y9. Telephone 902-473-4585, fax 902-473-5548, e-mail gswillia@dal.ca

Received for publication February 2, 2014. Accepted February 11, 2014 


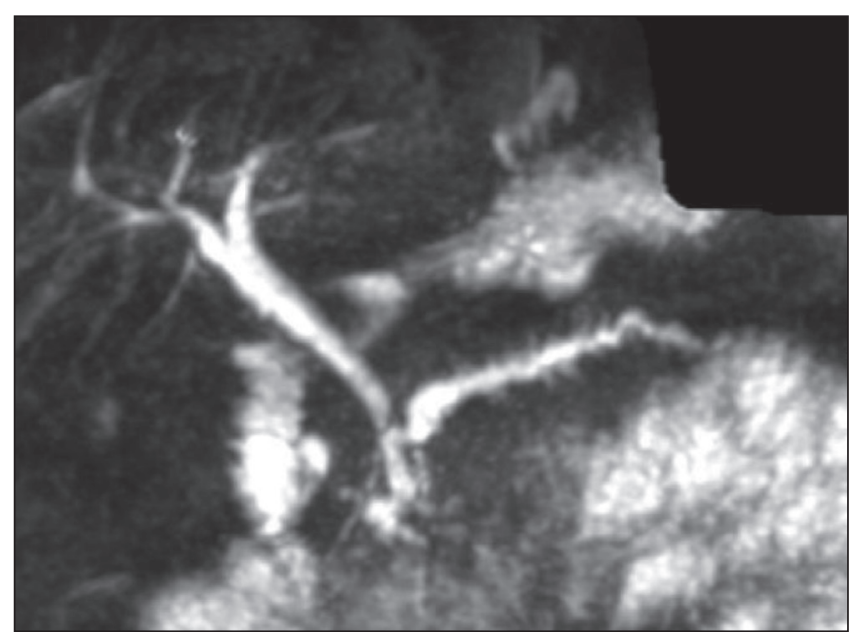

Figure 3) T2-weighted magnetic resonance image demonstrating cystic change in a thickened duodenal wall and progressive enhancement of soft tissue within the pancreatic duodenal groove. Magnetic resonance cholangiopancreatography demonstrates dilation and marked irregularity of the pancreatic duct and the tight, smooth stricture of the distal common bile duct

spleen-sparing distal pancreatectomy. Pathological analysis of resected specimens showed signs of inflammation with no indications of malignancy. The cyst in the pancreatic head was aspirated and cytology fluid was negative for malignant cells.

The patient experienced a smooth postoperative course until the in situ Jackson-Pratt drain was removed and the patient developed drainage from her wound. She was admitted to hospital and CT scan revealed a small peripancreatic collection with a new pancreaticocutaneous fistula. The patient was prescribed a proton pump inhibitor and subcutaneous octreotide and discharged with home care arranged.

\section{DISCUSSION}

GP is a form of segmental chronic pancreatitis characterized by fibrotic scarring of the gastroduodenal groove, an anatomical area bound by the pancreatic head, duodenum and CBD (1). It was first described by Potet and Duclert (2) in 1970, but remains a largely unrecognized entity to most clinicians. The condition has been reported under several alternative names, including paraduodenal pancreatitis, cystic dystrophy of heterotopic pancreas, paraduodenal wall cyst and myoadenomatosis (1).

In 1991, Becker and Mischke (3) identified two forms of GP - a pure form and a segmental form. Pure GP occurs solely within the gastroduodenal groove and spares the pancreatic parenchyma and main pancreatic ducts (3). Segmental GP affects both the gastroduodenal groove and pancreatic head, with stenosis of the pancreatic duct leading to proximal dilation (3).

GP presents most commonly in men in their fifth decade of life with a history of heavy alcohol use (1). Typical symptoms include postprandial epigastric pain, nausea, vomiting and weight loss $(1,3)$.

The current literature suggests that GP is a relatively common form of chronic pancreatitis. However, this diagnosis is not typically made preoperatively. In many of the previously reported cases, GP was not distinguished from pancreatic head carcinoma until after pancreaticoduodenectomy was performed and histological examination confirmed the diagnosis (4). The prevalence of GP among patients receiving pancreaticoduodenectomy for chronic pancreatitis has been reported to be as high as $24.5 \%$ (3).

Radiological imaging can be diagnostic for GP $(4,5)$, but depends on expert review of imaging, familiarity with the condition and

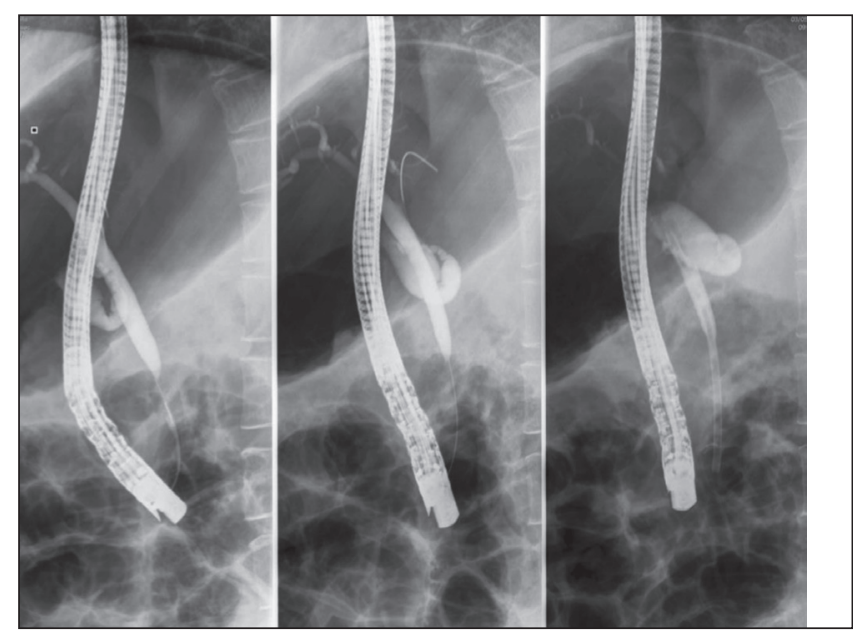

Figure 4) Images from endoscopic retrograde cholangiopancreatography demonstrating the smooth, tapered nature of the distal common bile duct stricture (in contrast to the abrupt 'cut-off' which is most often indicative of a malignant stricture)

communication of suspicion of the diagnosis with clinicians, as the present case illustrates. GP typically presents on CT or MRI as a poorly enhancing mass (6). Ductal dilation is uncommon, distinguishing it from adenocarcinoma (6). Contrast-enhanced CT characteristically reveals multiple cysts within a thickened duodenal wall and lesion enhancement following contrast injection due to associated inflammatory changes $(7,8)$. Small, intralesional ducts or cysts may be present and best appreciated by magnetic resonance pancreatography (9).

In summary, the current report presents an atypical presentation of GP in which diagnosis was made preoperatively by MRI, but was delayed due to lack of recognition of the features of the condition on initial imaging assessment. Greater clinical familiarity with GP should help to prevent excessive surgical intervention and better inform optimal management of this condition.

\section{REFERENCES}

1. Levenick JM, Gordon SR, Sutton JE, Suriawinata A, Gardner TB.

A comprehensive, case-based review of groove pancreatitis. Pancreas 2009;38:e169-75

2. Potet F, Duclert N. Cystic dystrophy on aberrant pancreas of the duodenal wall. Arch Fr Mal App Dig 1970;59:223-38.

3. Becker V, Mischke U. Groove pancreatitis. Int J Pancreatol 1991;10:173-82.

4. Malde DJ, Oliveira-Cunha M, Smith AM. Pancreatic carcinoma masquerading as groove pancreatitis: Case report and review of literature. JOP 2011;12:598-602.

5. Manzelli A, Petrou A, Lazzaro A, Brennan N, Soonawalla Z, Friend P. Groove pancreatitis. A mini-series report and review of the literature. JOP 2011;12:230-3.

6. Itoh S, Yamakawa K, Shimamoto K, Endo T, Ishigaki T. CT findings in groove pancreatitis: Correlation with histopathological findings. J Comput Assist Tomogr 1994;18:911-5.

7. Blasbalg R, Baroni RH, Costa DN, Machado MC. MRI features of groove pancreatitis. AJR Am J Roentgenol 2007;189:73-80.

8. Coakley FV, Hanley-Knutson K, Mongan J, Barajas R, Bucknor M, Qayyum A. Pancreatic imaging mimics: Part 1, imaging mimics of pancreatic adenocarcinoma. AJR Am J Roentgenol 2012;199:301-8.

9. Thomas H, Marriott P, Portmann B, Heaton N, Rela M.

Cystic dystrophy in heterotopic pancreas: A rare indication for pancreaticoduodenectomy. Hepatobiliary Pancreat Dis Int 2009;8:215-7. 


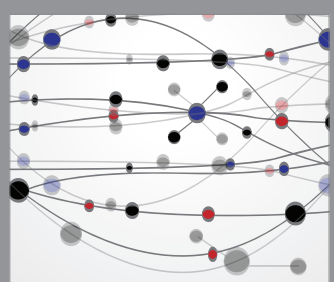

The Scientific World Journal
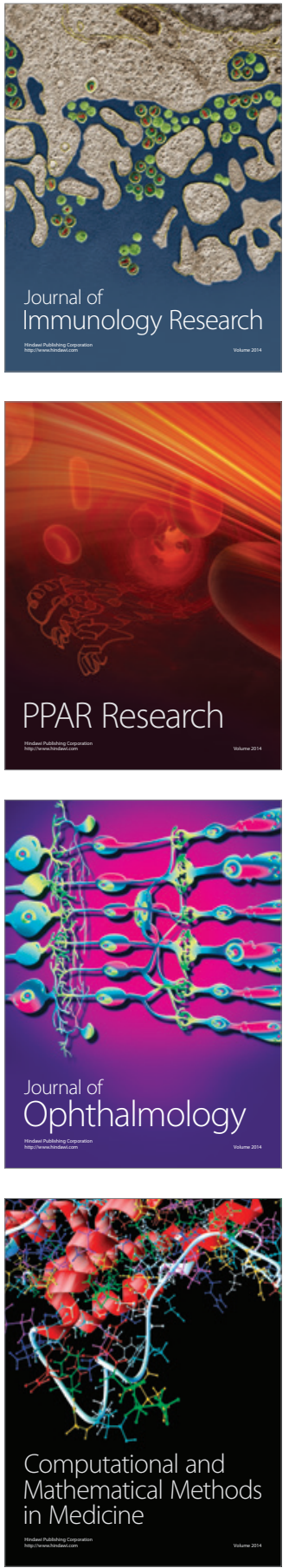

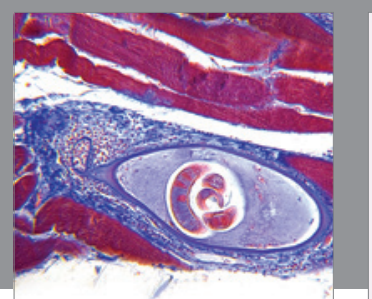

Gastroenterology Research and Practice

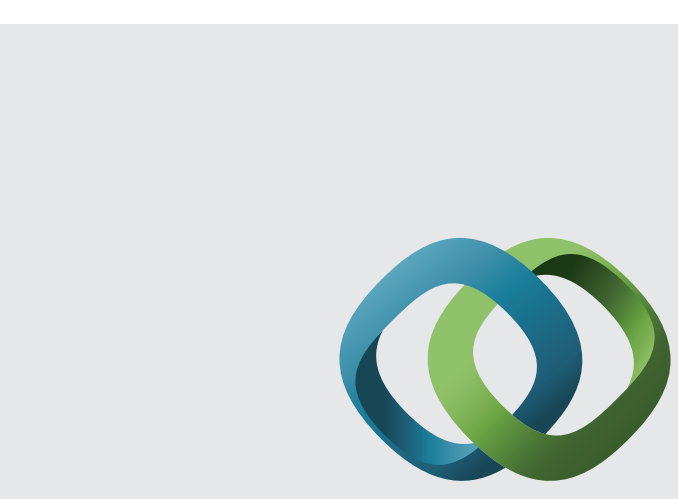

\section{Hindawi}

Submit your manuscripts at

http://www.hindawi.com
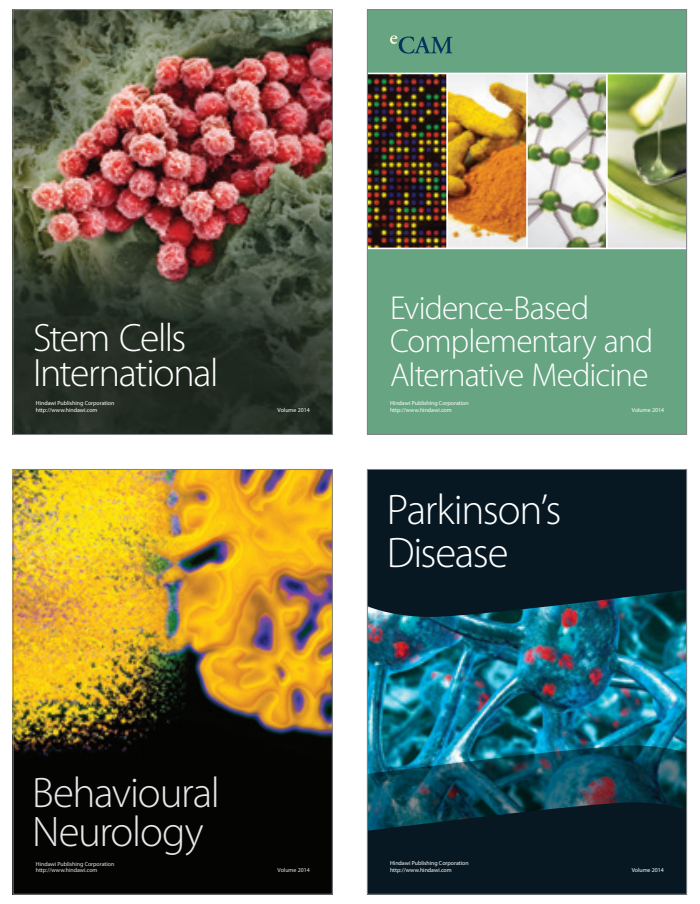
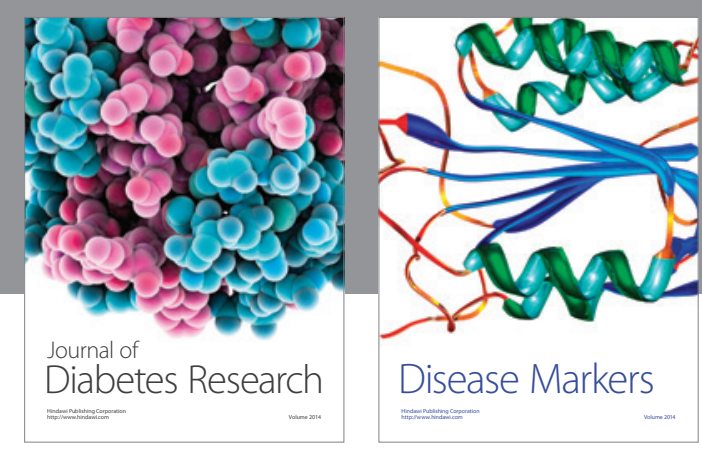

Disease Markers
